\title{
OTIMIZAÇÃO DE ESPAÇO FÍSICO EM UNIDADE DE ALIMENTAÇÃO E NUTRIÇÃO (UAN) CONSIDERANDO AVANÇOS TECNOLÓGICOS NO SEGMENTO DE EQUIPAMENTOS
}

\section{FOOD SERVICE LAYOUT OPTIMIZATION CONSIDERING EQUIPMENT TECHNOLOGICAL ADVANCES}

\author{
José Ramos Campos ${ }^{1}$ \\ Vivian Ikeda ${ }^{1}$ \\ Mônica Glória Neumann Spinelli
}

RESUMO: De acordo com a estimativa da Associação Brasileira das Indústrias dos Alimentos (ABIA, 2009), cerca de $25 \%$ das pessoas no Brasil tomam refeições fora de casa. O mercado de alimentação demonstra progressivo crescimento, apresentando para as empresas o desafio de oferecer serviços de qualidade. Para assegurar a excelência dos serviços prestados pelas Unidades de Alimentação e Nutrição (UANs), é imprescindível nortear o trabalho por meio das Boas Práticas de Fabricação (BPF). Entre as medidas apontadas pelas BPF, destaca-se a estrutura física das UANs, pois ela interfere na produção final do alimento, proporcionando ambiente adequado para a realização de um bom trabalho. Desse modo, o objetivo deste trabalho é comparar o espaço físico de uma UAN, levando em consideração o avanço tecnológico disponível no segmento de equipamentos. Quanto à metodologia empregada, foi realizado um estudo transversal, analisando o espaço físico de uma UAN que utiliza equipamentos convencionais, comparado com um Projeto de UAN para o mesmo local, utilizando equipamentos mais sofisticados. No estudo, houve uma comparação das dimensões das áreas atuais da UAN com um projeto de otimização do espaço físico. Os resultados identificam que o uso de equipamentos com avanços tecnológicos permite a construção de cozinhas industriais mais eficazes, com redução de espaço físico e melhorias nos processos de trabalho.

Palavras-chave: Unidade de Alimentação e Nutrição; espaço físico; equipamentos.

ABSTRACT: According to Brazilian Food Industries Association, about 25\% of Brazilian people have their meals way from home. The market for food services has demonstrated progressive growth, which makes it a challenge for companies to provide quality services. Good manufacturing practices (GMP) are essential to ensure excellent food services. Among measures proposed by GMP, the layout of food services interferes in food production providing an appropriate environment for a good job. Thus, the objective of this work was to compare the layouts of food services, taking into consideration technological advances available in this segment. A cross-sectional study was conducted that compared the layouts of two food services of the same unit, the first with conventional equipment and the second with more technological equipment. The measurements of the two layouts were also compared. The results indicate that the use of technological equipment allows the construction of more effective industrial kitchens with physical space reduction and improvements in work processes.

Keywords: food service; layout; equipment.

\footnotetext{
${ }^{1}$ Graduando(a) em Nutrição - Universidade Paulista - UNIP. E-mails: 12085@puras.com.br; vivian.ikeda@hotmail.com.

${ }^{2}$ Doutora em Saúde Pública - Universidade de São Paulo - USP e Docente da Universidade Presbiteriana Mackenzie - UPM e UNIP. E-mail: spinelli@usp.br.
} 


\section{INTRODUÇÃO}

De acordo com a estimativa da Associação Brasileira das Indústrias dos Alimentos (ASSOCIAÇÃO BRASILEIRA DAS INDUSTRIAS DE ALIMENTOS, 2009), cerca de $25 \%$ das pessoas no Brasil tomam refeições fora de casa. O mercado de alimentação demonstra progressivo crescimento, apresentando para as empresas o desafio de oferecer serviços de qualidade.

O mercado de alimentação é dividido em: alimentação comercial e alimentação coletiva, sendo que os estabelecimentos que produzem e distribuem alimentos para coletividades recebem, atualmente, o nome de Unidades de Alimentação e Nutrição (UANs) (VAZ, 2003; ABREU; SPINELLI; PINTO, 2011).

Uma UAN consiste em um serviço organizado, compreendendo uma sequência e sucessão de atos destinados a fornecer refeições balanceadas dentro dos padrões dietéticos e higiênicos sob o ponto de vista sanitário. Visa, ainda, a atender às necessidades nutricionais de seus clientes, de modo a se ajustar aos limites financeiros da instituição (ABREU; SPINELLI; PINTO, 2011).

Para assegurar a excelência do serviço, as UANs devem se preocupar com todas as etapas do processo, não apenas com a qualidade do alimento já pronto, mas com todos os fatores que podem interferir na qualidade da preparação durante 0 fornecimento da matéria-prima, no armazenamento e na sua produção (MARCON, 1997; BASTOS, 1999). O acesso às informações faz com que os consumidores fiquem mais atentos $e$ procurem estabelecimentos que ofereçam maior segurança $e$ atendam às suas expectativas (TEIXEIRA; OLIVEIRA; REGO, 2000; ANDRADE; SILVA; BRABES 2003).
Para Ribeiro (2003), devido ao grande fenômeno da globalização, para sobreviver à elevada competitividade e à questão da qualidade, as empresas foram obrigadas a se readequarem, visto a incompatibilidade apresentada pelas empresas com as Boas Práticas de Fabricação (BPF). Surgiu, a partir daí, a necessidade de identificar os problemas existentes e aplicar ações corretivas, que são um conjunto de medidas que a UAN deve adotar, baseando-se nas BPF, a fim de garantir a qualidade sanitária e manter a conformidade dos produtos alimentícios com os regulamentos técnicos (BRASIL, 2006).

As BPF são normas e procedimentos definidos previamente para atingir o padrão de qualidade de um serviço, pois a sua utilização requer uma avaliação periódica da qualidade higiênico-sanitária (CARDOSO, 1996; TEIXEIRA; OLIVEIRA; REGO, 2003). Nesse contexto, pode-se citar, como ponto chave para a implantação das BPF, a estrutura física das UANs, pois ela interfere na produção final do alimento, além de proporcionar um ambiente mais adequado para realizar um bom trabalho (TEIXEIRA; OLIVEIRA; REGO, 2003; MONTE, 2004, SANT'ANA, 2012).

Segundo Arruda (2002), é de extrema importância a participação de profissionais qualificados, como nutricionistas, na etapa do planejamento físico de uma UAN, pois possuem experiência tanto na área administrativa quanto na operacionalização das atividades de um restaurante. Proença (2003) cita que as instalações devem ser projetadas de forma a facilitar os procedimentos operacionais por meio de fluxos contínuos, sem o cruzamento de etapas e linhas do processo de produção, além de satisfazer o comensal com o serviço oferecido. Isso engloba desde o ambiente 
físico, incluindo tipo, conveniência e condições de higiene de instalações e equipamentos disponíveis, até o contato pessoal entre funcionários da UAN e os clientes, nos mais diversos momentos.

A análise da estrutura física e funcional de uma UAN é de fundamental importância para que se possam determinar as ações a serem tomadas e, assim, evitar eventuais desperdícios e problemas operacionais (TEIXEIRA; OLIVEIRA; REGO, 2003; GUIMARÃES, 2006).

Lúcia e Sant'Ana (2012) referem que uma UAN tem como objetivo satisfazer o cliente em relação ao serviço oferecido, englobando, sob esse aspecto, a estrutura física destinada aos clientes.

Visto que a literatura apresenta pouca produção sobre a interferência da tecnologia no planejamento físico de UANs, há a necessidade de fazer maiores estudos nessa área.

\section{OBJETIVO}

Comparar o espaço físico de uma Unidade de Alimentação e Nutrição convencional com um novo projeto para o mesmo espaço utilizando novas tecnologias de equipamentos.

\section{MÉTODO}

Trata-se de um estudo transversal, realizado no período de 05 a 22 de junho de 2011, em São Bernardo do Campo, SP.

Foi analisado o espaço físico de uma Unidade de Alimentação e Nutrição que utiliza equipamentos convencionais, comparado com um novo projeto para o mesmo local, que, por sua vez, utilizará equipamen- tos mais sofisticados (fornos combinados, frigideira elétrica, pistas de distribuição).

Foi utilizada trena para obtenção das medidas referentes aos equipamentos e espaço físico, e o programa Excel para tabulação dos dados. Foram medidos todos os equipamentos da cozinha convencional, assim como os equipamentos previstos no projeto.

Foram feitas comparações dos dados do projeto em relação à UAN estudada, traçando um paralelo com a Norma Regulamentadora 24 (NR24), (BRASIL, 1978), que preconiza as medidas de UANs.

Para a realização da pesquisa, foi encaminhado um Termo de Consentimento para a empresa na qual está inserida a UAN e, após a explicação dos objetivos da pesquisa, foi solicitada a autorização para a realização do trabalho. Foram garantidos o sigilo do nome da empresa e a devolução dos resultados e análise dos dados encontrados.

O estudo retrata as condições ideais de trabalho para uma cozinha industrial que produz 1300 refeições por dia, servidas em três turnos (700 almoços, 350 jantares e 250 ceias), utilizando equipamentos avançados tecnologicamente, visando a otimizar o espaço físico com novas definições das áreas.

\subsection{Espaço Físico}

Para o cálculo da necessidade de espaço físico (em metros) de áreas específicas da UAN, foi proposto um espaço físico de circulação maior do que preconiza a NR24, pois, na prática, acredita-se que o espaço exigido por lei é insuficiente. 


\subsection{Estoque}

O espaço físico do estoque levou em consideração o tipo de cardápio, número de refeições servidas, gêneros utilizados, período de abastecimento e per capita, considerando o estoque mínimo de três dias.

Quanto ao cálculo dos alimentos, considerou-se o consumo dos alimentos em quilos, de acordo com o período de abastecimento, per capita e tipo de atividade dos comensais (operacionais, administrativos ou mistos).

No que diz respeito ao armazenamento, para cada fardo de 30 quilos de alimento foi estipulado um espaço de $0,42 \mathrm{~m}^{2}$, estimado como suficiente para armazenar com uma boa aeração em uma altura de 8 fardos.

\section{3 Área de Produção}

Para cálculo dessa área, pensou-se no binômio funcionário $x$ equipamentos. $O$ cálculo de funcionários foi baseado no mercado, ou seja, no número usado pelas principais concessionárias que compõem esse segmento. $\mathrm{O}$ cálculo do espaço físico dos equipamentos foi baseado em medidas dos equipamentos e informações dos Fornécedores, respeitando o espaço ideal para circulação de funcionários, com segurança.

\subsection{Normatização do restaurante}

A área do restaurante foi projetada considerando $80 \%$ do número de refeições de maior pico (conforme preconiza a NR24), porém com espaços de circulação, entre as mesas, maiores que os exigidos, por ser observado uma deficiência de espaço ao serem consideradas as recomendações da NR24.

\section{RESULTADOS E DISCUSSÃO}

Os resultados expressos na Tabela 1 mostram as diferenças entre a UAN estudada e o novo projeto para essa Unidade de Alimentação e Nutrição.

Tabela 1 - Dimensões das áreas $\left(m^{2}\right)$

\begin{tabular}{lccc}
\hline \multicolumn{1}{c}{ Áreas da UAN } & UAN estudada & Projeto & Diferença \\
\hline Copa de café & 09 & 06 & 03 \\
Restaurante & 646 & 420 & 226 \\
Distribuição & 39 & 20 & 19 \\
Higiene de louças & 51 & 22 & 29 \\
Estoque de material descartável & 20 & 09 & 11 \\
Sala do nutricionista & 22 & 10 & 12 \\
Higienização de panelas & 30 & 09 & 21 \\
Estoque de material de limpeza & 15 & 08 & 07 \\
Área de produção & 285 & 65 & 220 \\
Estoque seco e refrigerado & 49 & 35 & 14 \\
Central de gás & 10 & 05 & 05 \\
Área de Pré-preparo & 16 & 10 & 06 \\
Área de recebimento & 20 & 15 & 05 \\
Vestiário feminino & 19 & 15 & 04 \\
Vestiário Masculino & 32 & 24 & 08 \\
Câmara de lixo & 15 & 07 & 08 \\
Total & 1278 & 680 & 598 \\
\hline
\end{tabular}

Fonte: SBC (2011). 
A Fig. 1 retrata uma UAN cocção. Com o uso de equipamentos mais convencional, ou seja, sem o uso de avançados, percebe-se uma redução equipamentos tecnologicamente avançados. significativa no número de equipamentos, Identifica-se grande quantidade de funcionários e otimização de espaço físico equipamentos e, também, maior número de (conforme mostra a Fig. 2).

profissionais envolvidos no processo de
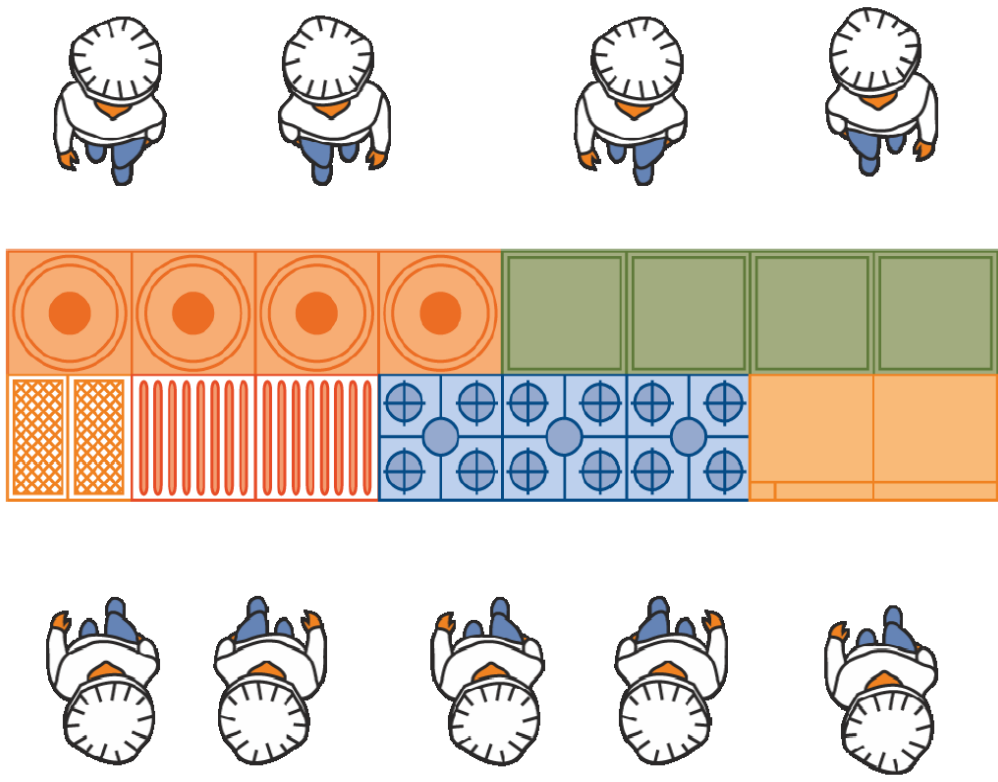

Fig. 1 - Espaço da UAN convencional. SBC, 2011.

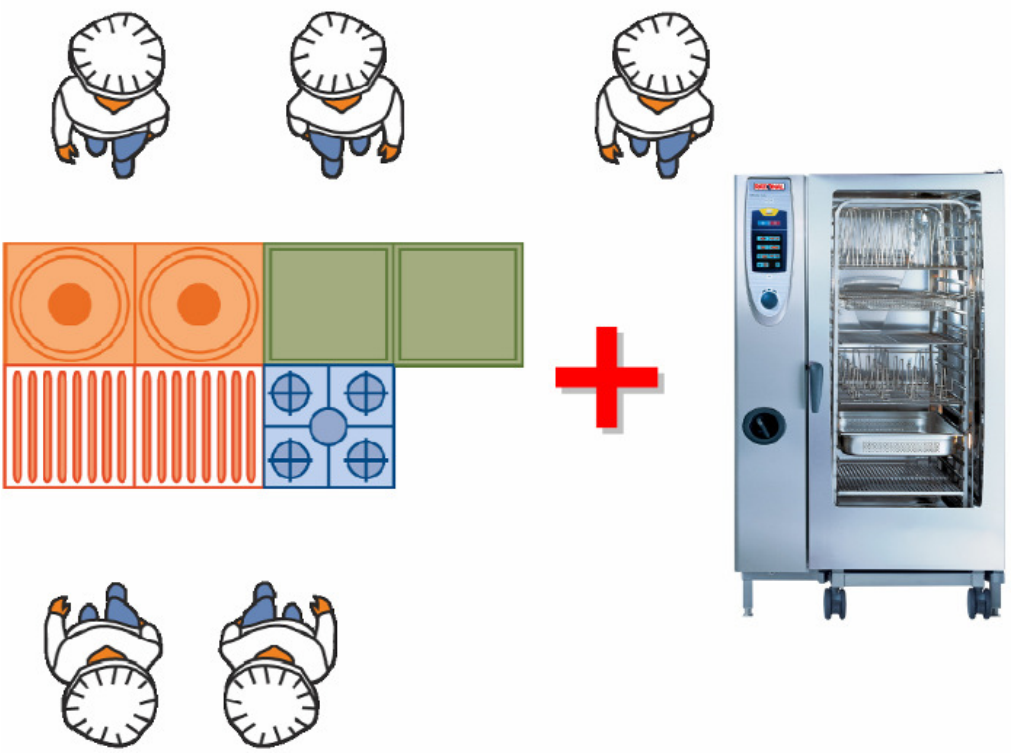

Fig. 2 - Espaço do projeto. SBC, 2011. 
Quadro 1 - Necessidade de equipamentos segundo o tipo de UAN.

\begin{tabular}{lccc}
\hline \multicolumn{1}{c}{ Equipamentos } & $\begin{array}{c}\text { UAN } \\
\text { estudada }\end{array}$ & Projeto & $\begin{array}{c}\text { Redução em } \\
\%\end{array}$ \\
\hline Caldeirões & 4 & 2 & 50 \\
Frigideiras basculante & 4 & 2 & 50 \\
Chapeiras & 2 & 0 & 100 \\
Grill & 2 & 2 & 0 \\
Fogões de 4 bocas & 3 & 1 & 66 \\
Fritadeiras para 180 litros de óleo & 2 & 0 & 100 \\
\hline
\end{tabular}

Fonte: SÃO PAULO (2011).
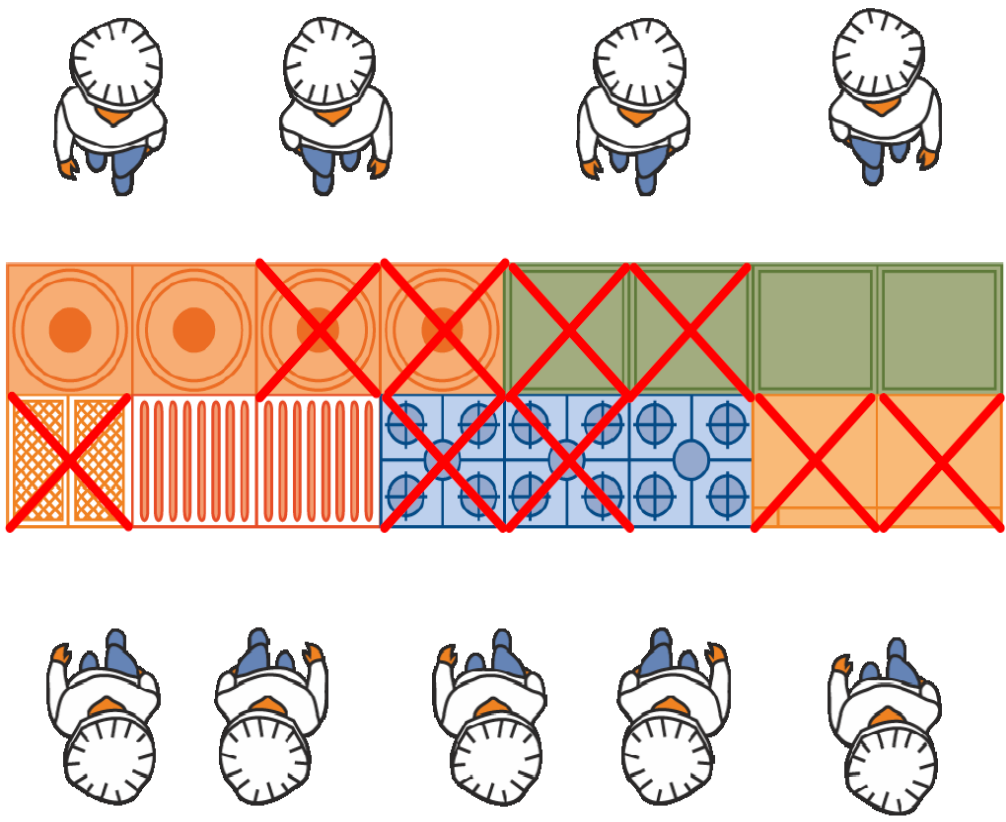

Fig. 3 - Mostra as alterações na UAN tecnologicamente equipada. SBC, 2011.

A Fig. 3 destaca a eliminação de equipamentos convencionais mediante a substituição por equipamentos de alta tecnologia.

No novo projeto para a UAN estudada, recomenda-se o uso de fornos combinados. Estes medem $160 \mathrm{~cm}$ de altura, $120 \mathrm{~cm}$ de profundidade e $90 \mathrm{~cm}$ de largura. Os fornos combinados substituem os seguintes equipamentos: duas caldeiras, duas frigideiras, dois fogões, duas fritadeiras e duas chapeiras. A substituição desses equipamentos totalizam uma economia de $10,36 \mathrm{~m}^{2}$ de espaço físico.

Neste estudo, identificou-se que a legislação subestima a área real necessária para estoque e produção, conforme também apontam Sant'Ana e Campos (2012).

O novo projeto para a UAN estudada contempla uma área de circulação maior do que preconiza a NR24, pois foi observado que o espaço proposto pela norma é insuficiente. Desse modo, a área prevista para circulação passa a ser de $1,20 \mathrm{~m}$ a 
1,50 m entre os equipamentos. Os espaços observados na UAN são insuficientes para uma circulação segura (distância de 0,75 m entre bancadas e 0,55 $\mathrm{m}$ de área para circulação).

\section{Quanto ao recebimento}

A área de recebimento é o local destinado à recepção de todas as mercadorias que chegam à UAN. Deve apresentar espaço e equipamentos necessários para um bom atendimento, suprindo as necessidades do estoquista, como balanças, carros de transporte de mercadoria, mesa de apoio, computador para o controle de entrada de notas, calculadoras e outros. Essa área apresentava um bom dimensionamento na UAN estudada, mas ainda foi possível reduzir $5 \mathrm{~m}^{2}$ no novo projeto, conforme pode ser observado na Tabela 1. Segundo Sant 'Ana e Azeredo (2012), a garantia da qualidade das refeições e o controle de custos em uma UAN dependem das condições estruturais dessa área, para que as matérias primas sejam recebidas e inspecionadas adequadamente.

\section{Quanto ao armazenamento}

Para a área destinada ao estoque de material descartável, foram calculados $9 \mathrm{~m}^{2}$, e alocadas 6 estantes de aço inox de 100 $\mathrm{cm} \times 70 \mathrm{~cm}$, sobrando espaço para a circulação do ar.

A área reservada para estoque seco e refrigerado foi de $35 \mathrm{~m}^{2}$. Nessa área, foram locadas 10 estantes de aço inox, medindo $170 \mathrm{~cm}$ x $90 \mathrm{~cm}, 15$ paletes de $90 \mathrm{~cm} \times 90$ $\mathrm{cm}, 1$ mesa de computador, $120 \mathrm{~cm} \times 90 \mathrm{~cm}$. Com o espaço físico reservado para essa área alocaram-se todos os mobiliários, respeitando um espaço mínimo de $10 \mathrm{~cm}$ entre paletes e estantes, além da altura mínima de $5 \mathrm{~cm}$ exigida pela CVS6/99. O espaço foi elaborado baseado no cardápio e no volume de compra/dia praticado pela maioria das concessionárias desse segmento, respeitando a compra de duas a três vezes por semana, para melhor controlar a gestão. Segundo Sant 'Ana e Azeredo (2012) e a RDC 275 (BRASIL, 2002), é recomendável que a entrega de matéria-prima seja feita com a maior frequência possível, requerendo menor espaço para estocagem e diminuição com os custos da construção.

\section{Quanto à área de produção}

A área de pré-preparo é destinada ao corte de carnes e higiene de frutas verduras e legumes (FLV). Há uma obstrução física separando a área de carne da área de FLV.

Segundo Sant 'Ana e Azeredo (2012), sempre que possível, devem ser utilizados equipamentos que agreguem tecnologia, a fim de diminuir a quantidade de equipamentos e ganhar produtividade.

No dimensionamento da cozinha (área de produção), é possível realizar uma otimização do espaço, fazendo uso de equipamentos de alta tecnologia (fornos combinados, passthrough, caldeiras, frigideiras basculantes, chapeira elétrica, máquina de lavar louças, tubo de secagem, carrinhos de transportes e outros).

Foram analisados os espaços físicos, de cada área, levando em consideração a dimensão dos equipamentos:

$\mathrm{Na}$ copa de café, no novo projeto, é utilizada uma máquina para o preparo de 
café, com a dimensão de $200 \mathrm{~cm}$ x $90 \mathrm{~cm}$ e $120 \mathrm{~cm}$ de altura, com dois compartimentos de 70 litros cada. Foram dimensionadas, ainda, uma pia de $100 \mathrm{~cm} \times 60 \mathrm{~cm}$ e uma cuba de inox de $50 \mathrm{~cm} \times 80 \mathrm{~cm}$ e $90 \mathrm{~cm}$ de profundidade, para higiene dos coadores e partes móveis da máquina e uma mesa de apoio de $160 \mathrm{~cm} \times 80 \mathrm{~cm}$. Mesmo com os equipamentos instalados, nota-se que há espaço físico ideal para a circulação dos funcionários.

A área de produção foi projetada para acomodar todos os equipamentos da cozinha, conforme descrição abaixo:

Dois fornos combinados de $120 \mathrm{~cm} \mathrm{x}$ $90 \mathrm{~cm}$, com capacidade para 42 GNs (gastronormes); 1 fogão de 04 bocas, de $120 \mathrm{~cm} \times 160 \mathrm{~cm} ; 1$ frigideira basculante, de $90 \mathrm{~cm} \times 90 \mathrm{~cm} ; 2$ caldeiras a vapor, com capacidade de 500 litros cada, medindo 150 $\mathrm{cm} \times 150 \mathrm{~cm}$; 1 batedeira industrial, de $90 \mathrm{~cm}$ x $90 \mathrm{~cm}$; 1 chapeira elétrica, de $60 \mathrm{~cm}$ x 120 $\mathrm{cm}$; 5 mesas de apoio em aço inox, de 120 $\mathrm{cm} \times 90 \mathrm{~cm}$; 2 geladeiras industriais, de 220 $\mathrm{cm} \times 90 \mathrm{~cm} ; 1$ freezer, de $140 \mathrm{~cm} \times 90 \mathrm{~cm} ; 1$ bebedouro, de $60 \mathrm{~cm} \times 60 \mathrm{~cm} ; 1$ fritadeira, de $160 \mathrm{~cm}$ x $80 \mathrm{~cm} ; 2$ char broilers, $90 \mathrm{~cm} \times$ $70 \mathrm{~cm}$. O projeto deve locar os equipamentos de maneira que facilite o fluxo de pessoas e não acarrete insegurança, ou seja, riscos de acidentes, como impacto das pessoas contra os equipamentos, principalmente quando estiverem em funcionamento ou com as portas abertas.

Os passthroughs são os únicos equipamentos de uma UAN que não ocupam tanto espaço físico. Como o próprio nome já diz, esse equipamento proporciona uma passagem dos alimentos de um lado para outro. Esse equipamento tanto pode ser aquecido como refrigerado, são locados geralmente em um espaço planejado, embutido na parede que separa a cozinha do restaurante. Os alimentos armazenados nesses equipamentos são preparados e colocados "na espera" para abastecer a área de distribuição.

\section{Quanto à área de distribuição}

O restaurante é uma área pela qual há uma grande preocupação das empresas de refeições, não apenas com o layout, mas também com o bem-estar e comodidade dos consumidores, visto que houve uma preocupação no espaço entre as mesas de, aproximadamente, 1,2 m. Para atender os usuários, foi prevista uma área de $220 \mathrm{~m}^{2}$, para acomodar 140 mesas, de $120 \mathrm{~cm} \times 70$ $\mathrm{cm}$, com espaçamento de 1,2 m. A NR24 (BRASIL, 1978) estabelece que locais com mais de 300 funcionários devem possuir um salão de refeições com, no mínimo, $1 \mathrm{~m}^{2}$ por usuário, podendo abrigar, de cada vez, um terço dos funcionários. Essa indicação não considera o fluxo real de clientes, podendo resultar em uma área sub ou super dimensionada. Foi observado que 0 espaçamento preconizado pela NR 24 (BRASIL, 1978) é de $0,75 \mathrm{~m}$ na circulação principal e 0,55 m entre as mesas. Não é um espaçamento confortável e nem seguro para a circulação de duas pessoas com bandejas nas mãos.

A área de distribuição é um local que requer um espaço bem planejado. $O$ funcionário que abastece as pistas quentes e frias divide o espaço com os clientes (usuários), e, por isso, há um risco de acidentes elevadíssimo. Quando esse espaço é bem planejado, há uma diminuição nos riscos, não basta ter espaço físico em abundância e um layout que não atenda às necessidades da UAN, em relação à praticidade e segurança. É de extrema 
importância que o espaço seja bem planejado para evitar choque entre os clientes usuários e, até mesmo, com os funcionários da UAN e, consequentemente, causar acidentes. O planejamento desse espaço deve contemplar a medida dos utensílios e do mobiliário, a disposição das mesas e das cadeiras, o número de clientes a serem atendidos, os horários disponíveis para a refeição e tipo de fluxo dos clientes (SANT'ANA; AZEREDO, 2012).

Quanto ao local de higienização e tratamento do lixo

A área de higiene de louças fica, geralmente, restrita a um canto qualquer que as empresas estipulam para um funcionário trabalhar durante 8 horas por dia. É importante lembrar que essa área é o local menos desejado de toda cozinha, ou seja, menos aceito. Foi sugerido uma área de 22 $\mathrm{m}^{2}$; com uma bancada e pias de inox, medindo, no total, $300 \mathrm{~cm} \times 100 \mathrm{~cm} ; 4$ estantes de inox, de $210 \mathrm{~cm}$ x $80 \mathrm{~cm} ; 4$ tambores de lixo, de 200 litros, para separação dos lixos; e 2 mesas de apoio, de $200 \mathrm{~cm}$ x $90 \mathrm{~cm}$, para apoio das panela antes da higienização. Mesmo com todos mobiliários sobra espaço físico para a locomoção do profissional que trabalha na área e acesso de outros funcionários.

A câmara de lixo foi programada para armazenar restos de alimento somente de um dia.

\section{Anexos}

A sala do nutricionista foi projetada com $10 \mathrm{~m}^{2}$ para comportar 2 mesas de computadores, de $150 \mathrm{~cm} \times 90 \mathrm{~cm}$, e uma mesa de apoio, de $120 \mathrm{~cm}$ x $90 \mathrm{~cm}$, sobrando espaço suficiente para cadeiras para visitas.

A área reservada para os recipientes que armazenam o gás faz parte da UAN, porém deve ficar situada fora da cozinha, necessita apenas do espaço para a guarda dos tanques e espaço para circulação do funcionário da empresa que abastece o gás. Não necessita dispor de um espaço físico grande.

Os vestiários foram construídos respeitando a NR24 (BRASIL, 1978), que preconiza $1,5 \mathrm{~m}^{2}$ para cada funcionário. No quadro desse projeto, serão 20 funcionários, contando com o nutricionista, sendo $40 \%$ do sexo feminino e $60 \%$ masculino.

\section{CONCLUSÃO}

Conclui-se que o uso de equipamentos com avanço tecnológico permite a construção de cozinhas industriais modernas e confortáveis para trabalhar, com redução de espaço físico.

Pode-se concluir, ainda, que é difícil a aplicação de índices que atendam a todos os projetos, ou seja, cada um requer um estudo individualizado, considerando as particularidades e a organização do serviço de cada UAN.

\section{REFERÊNCIAS}

ASSOCIAÇÃO BRASILEIRA DAS INDUSTRIAS DE ALIMENTOS. Alimentos fora do lar, 2009. Disponível em: http://www.abia.org.br/sp. Acesso em: 25 maio 2011.

ANDRADE, N. J.; SILVA, R. M. M.; BRABES, K. C. S. Avaliação das condições microbiológicas em unidade de alimentação 
e nutrição. Ciênc. Agrotec., 2003, v. 27, n. 3, 590-596.

ABREU, E. S.; SPINELLI, M. G. N.; SOUZA PINTO, A. M. P. Gestão de Unidades de Alimentação e Nutrição: um modo de fazer. 4. ed. São Paulo: Metha, 2011.

ARRUDA, G. A. Manual de Boas Práticas: Unidades de Alimentação e Nutrição. 2. ed. São Paulo: Ponto Crítico, 2002, v. 2, p. 234236.

BASTOS, M. S. R. Leite longa vida UHT: Aspectos do processamento e identificação dos pontos críticos de controle. Revista Higiene Alimentar, São Paulo, v. 13, n. 66/67, p. 32-36, 1999.

BRASIL. Agência Nacional de Vigilância Sanitária. Resolução RDC 275 de 21/10/2002: Regulamento técnico de procedimentos operacionais padronizados aplicados aos estabelecimentos produtores/industrializadores de alimentos. Diário Oficial da União, Brasília, 6/9/2002. Ministério da Saúde. Secretaria de Políticas de Saúde. Protocolo de Investigação, diagnóstico, tratamento e prevenções de lesões por esforços repetitivos: distúrbios osteomusculares relacionados ao trabalho. Brasília: (MS), 2006.

Secretaria de Segurança e Medicina do Trabalho. Portaria G. M. no 3214 de 08/06/1978. Condições Sanitárias e de Conforto nos Locais de Trabalho. Diário Oficial da União, Brasília, 1978.

CARDOSO, R. C. V. et al. Avaliação da eficiência da agencia sanitária para mãos de manipuladores de alimentos em serviços de refeição coletiva. Higiene alimentar, v. 10 p. 17-22. 1996.

GUIMARÃES, I. A. A análise da estrutura física e funcional de uma UAN é de fundamental importância para que se possam determinar as ações a serem tomadas e assim evitar eventuais desperdícios e problemas operacionais. 2006. 69p. Dissertação (Mestrado) Universidade de Brasília, Centro de Excelência em Turismo, Brasília, 2006.

LUCIA, C. M. D.; SANT'ANA, H. M. P. Introdução ao Planejamento Físico de Unidades de Alimentação e Nutrição in SANT'ANA, H. M. P. Planejamento físico funcional de Unidades de Alimentação e Nutrição. Rio de Janeiro: Rubio, 2012. p.1-9.

MARCON, M. C. As novas propostas de organização do trabalho e a participação do trabalhador: um estudo de caso, desenvolvido junto a uma unidade de alimentação e nutrição tipo concessionária, sob enfoque ergonômico. 1997. 108 p. Dissertação (Mestrado em Engenharia) Programa de Pós Graduação em Engenharia de Produção, Universidade de Santa Catarina, Florianópolis.

MONTE, C. R. V. et. al. Aplicação de um modelo para avaliar projetos de unidades de alimentação e nutrição. Nutrição Brasil. São Paulo, v. 3, n. 2, p. 11-17, jan./fev. 2004.

PROENÇA, R. P. C. Ergonomia e organização do trabalho em projetos industriais: uma proposta no setor de Alimentação Coletiva. 1993. Dissertação (Mestrado). Universidade Federal de Santa Catarina, Florianópolis, 1993.

RIBEIRO, C. da S. G. Controle de custos: questão de sobrevivência para as Unidades de Alimentação e Nutrição. Revista Nutrição Brasil, jan./fev. 2003, v. 2, n. 1, p. 39-44.

SANT'ANA, H. M. P. Introdução ao Planejamento Físico de Unidades de Alimentação e Nutrição in SANT'ANA, H. M. 
P. Planejamento físico funcional de M. P. Planejamento físico funcional de Unidades de Alimentação e Nutrição. Rio de Unidades de Alimentação e Nutrição. Rio de Janeiro: Rubio, 2012. p.1-9. Janeiro: Rubio, 2012. p. 85-109.

SANT'ANA, H. M. P.; AZEREDO, E. M. C. TEIXEIRA, S. M. F. G.; OliVeIRA, Z. M. C.; Planejamento dos Setores de Distribuição, REGO, J. C. Administração aplicada às Consumo, Higienização e Pessoal. In: unidades de alimentação e nutrição. São SANT'ANA, H. M. P. Planejamento físico Paulo: Atheneu, 2003.

funcional de Unidades de Alimentação e Nutrição. Rio de Janeiro: Rubio, 2012. p.135-168.

VAZ, C. S. Alimentação de coletividade. Uma abordagem regional. 2. ed. Brasília: Metha, 2003.

SANT'ANA, H. M. P.; CAMPOS, F. M. Dimensionamento dos Setores da Unidade de Alimentação e Nutrição in SANT'ANA, H. 